\title{
Undue Hardness/Modulus Ratio Claims instead of Physical Penetration Resistance and Applications with Mollusk Shells
}

\author{
Gerd Kaupp \\ University of Oldenburg, Oldenburg, Germany \\ Email: gerd.kaupp@uni-oldenburg.de
}

How to cite this paper: Kaupp, G. (2021) Undue Hardness/Modulus Ratio Claims instead of Physical Penetration Resistance and Applications with Mollusk Shells. $A d$ vances in Materials Physics and Chemistry, 11, 45-57.

https://doi.org/10.4236/ampc.2021.112005

Received: February 1, 2021

Accepted: February 25, 2021

Published: February 28, 2021

Copyright $\odot 2021$ by author(s) and Scientific Research Publishing Inc. This work is licensed under the Creative Commons Attribution International License (CC BY 4.0).

http://creativecommons.org/licenses/by/4.0/

\begin{abstract}
The Nanoindentation is a precise technique for the elucidation of mechanical properties. But such elucidation requires physically based interpretation of the loading curves that is widely still not practiced. The use of indentation hardness $H$ and indentation modulus $E_{\mathrm{r}}$ is unphysical and cannot detect the most important phase-transitions under load that very often occur. The claim that $H$ versus $E$ plots relate linearly for all different materials is neither empirically found nor correctly deduced. It is most dangerous by producing incorrect materials properties and misleading. The use of $H / E$ (that is also called "elasticity index") in complicated formulas for brittle parameter, yield strength, toughness, and so-called "true hardness" is also in error. The use of $H / E$ cannot reveal the true qualities of materials without considering phase-transitions under load that require the correct exponent $3 / 2$ on $h$ for the loading curves (instead of disproved 2). This is exemplified with the physical data of different mollusk shells that experience phase-transitions, a new bionics model, and different contributions for their strengthening. The data are compared to the ones of aragonite and calcite and vaterite.
\end{abstract}

\section{Keywords}

Nanoindentation, H/E Ratio Challenge, Phase-Transitions, Penetration

Resistance, Mollusk Shells, Aragonite, Calcite, Bionics

\section{Introduction}

The recent papers of Labonte, Lenz and Oyen [1] including hardness and moduli data from indentations onto calcite, aragonite, and nacre, and a multitude of other materials, or the one of Teniswood, Roberts, Howard and Bradby 
on the pyramidal nanoindentation onto aragonite of pteropod shells [2] are unphysical and burden with historical errors despite long known physical knowledge on the basis of simple arithmetic [3]. It is empirically known since 2004 [4] and 2013 with extensive table for all types of materials [5] and undoubtedly physically deduced one year before 2016 [3] and also in 2020 [6] that pyramidal and conical indentations follow the exponent $3 / 2$ on the depth $h$ (but not 2 ) in the force $\left(F_{\mathrm{N}}\right)$ vs depth curves, the slope of which is the penetration resistance, that is the physical hardness when calibrated with the indenter cone or effective cone. It was deduced in 2013 [7] and in 2017 [8] that the Oliver-Pharr iterations that are still ISO 14577 standard violate the energy law for hardness $H$ [7] and in 2017 [8] also for $E_{\mathrm{r}}$ since 2017 [9] (ISO denotes International Standardization Organization). Furthermore, elastic moduli from indentations are not "Young's moduli", as used in [1] and [2]. Both quantities should not rely on the three and eight free parameters iterations. And one does not know which of the polymorphs of the material under the applied force was probed. The detection of such polymorphism is only possible by using the $F_{\mathrm{N}}$ vs $h^{3 / 2}$ relation [3] [4] [5]. It is known since 2010 how unsteadiness kinks in $F_{\mathrm{N}}$ vs $h^{3 / 2}$ plots detect important phase transitions upon indentations in [10], in 2018 [11], and in numerous further publications of the present author. The calculation of phase-transition energies was developed in 2013 [7], in 2014 [12], in 2019 [13] [14], and in 2020 [15], the calculation of activation energies of phase transitions in 2014 [12].

But all of that has been disregarded by the criticized authors who refer to Oliver-Pharr [16] and prefer the corresponding errors and energy law violations rather than checking the exponent on $h$ of their loading curves (it is in all cases $3 / 2$ and not 2). The numerous undeniable listed applications are not at all available with the physically false assumed exponent. Such $H$ and $E_{\mathrm{r}}$ are fake-values and so are there from created theories of [1] and [2] that must be urgently criticized.

\section{Materials and Methods}

The published Berkovich indentation loading curves from the Limacina Helicina Antarctica and Haliotis rufescens mollusks were scanned and enlarged to A4 size. 25 to 42 data points were taken for the Excel calculation of $F_{\mathrm{N}}$ vs $h^{3 / 2}$ diagrams. The regression lines of the linear branches provide the slopes as $k$-values (the physical hardness) for the precise calculation of the kink position (phase-transition onset). These are the basis for the calculation of indentation work ( $W_{\text {indent }}$ ), applied work ( $\left.W_{\text {applied }}\right)$, full applied work (full $W_{\text {applied }}$ ) and phase-transition energy ( $\left.W_{\text {conversion }}\right)$, using a pocket calculator (10 digits before final rounding). We normalize them per $\mu \mathrm{N}$ to make them comparable. The necessary equations are well known as repeatedly published in [3] [6] [14], and earlier publications of the present author. The criticized log-log diagrams from [1] are checked for its missing validity even within the long disproved ISO- $H$ and ISO- $E_{\mathrm{r}}$ world. 


\section{Results ad Discussion}

\subsection{The Still Believed $H / E$ ("Elastic Index") Claims}

The methods of [1] are for various reasons questionable and useless. The authors do not explain why they claim proportionality between ISO- $H$ and ISO- $E_{\mathrm{r}}$ (here for Berkovich). The according to [16] defined ISO- $H$ is $F_{\mathrm{N}}$ per contact area $A_{\mathrm{hc}}$ that is geometrically $27.15 h_{c}{ }^{2}$. The dimension is (force/depth ${ }^{2}$ ) usually reported as GPa. The elastic property is experimentally measured as stiffness $S=$ $\Delta F_{\mathrm{Nmax}} / \Delta h$ with the different dimension (force/depth). To obtain an ISO- $E_{\mathrm{r}}$ with the same dimension as the ISO- $H$, one multiplies the stiffness $S$ with $0.5 \pi^{1 / 2}$ $A_{\mathrm{hc}}{ }^{-1 / 2}$. This provides the further $h_{\mathrm{c}}^{-1}$ for the dimension of ISO- $E_{\mathrm{r}}$ as force per area (GPa). But the $A_{\mathrm{hc}}$ value requires one iteration with 3 and another iteration with up to 8 free parameters (also + or - sign selection) according to [16]. The plots of $\log H$ vs $\log E_{r}$ numbers, suggesting a “ $\approx 0.05$ ratio of $H / E_{r}$ ” for uncountable published Berkovich indentations would at best indicate very poor worldwide measurements of indentations over the years if that would be reality. It cannot be used for the calculation of $E_{\mathrm{r}}$ from $H$ numbers with a "statistical confidence of $95 \%$ " and $\mathrm{R}^{2}=0.96$. For example a hardness number $H$ of $0.6 \mathrm{GPa}$ in figure 1 of [1] contains a spread from 6-30 GPa for the moduli $E_{\mathrm{r}}$ of the densely overlapping entries. Or an entry at $H=7 \times 10^{-5} \mathrm{GPa}$ has a data triangle value of about $1.05 \times 10^{-2} \mathrm{GPa}$ for $E_{r}$, while the corresponding $H$ value on the $H / E_{\mathrm{r}}$ line for that $E_{\mathrm{r}}$ is at $4.8 \times 10^{-4} \mathrm{GPa}$, which is an about 6.9-fold higher hardness number. These examples show drastically that the claimed linear relation between the ISO hardness $H$ and ISO modulus $E_{\mathrm{r}}$ numbers is not correct. And it will be shown in Section 3.2 why it cannot be correct. The claimed statistic confidence of $95 \%$ for the log-log plot is useless and dangerous. In the figures 1 and $4 \mathrm{a}, 4 \mathrm{~b}$ of [1] there must be selective choices of data from old papers and tables (often not the more recent ones) with questionable reliability. And even in the more recent papers it was never considered or known which polymorph of the sample had been probed under load, because their onsets could never been seen or excluded. We also must complain that the used entries in figure 1 of [1] are not cited and the materials not named. It appears that numerous of these are unpublished own values. This must also be concluded from the caption of figure 4 in [1], where, unlike the $H / E_{\mathrm{r}}$ plot, straight linear plots are imaged. These are for spherical and for Berkovich indentations without any visible deviations. Beware from the risk of $H$ vs $E_{\mathrm{r}}$ plots in view of figure 1 of [1] and beware from predictive uses from there!

Particularly risky and dangerous are the use of $H / E_{\mathrm{r}}$ plots or values for the evaluation of brittleness characterizations, critical load ratios, strengthening, toughness, and "true hardness". For example figure $4 \mathrm{a}$ in [1] describes linear correlations of the brittleness parameter against the load ratio, which rests on $\left(H / E_{\mathrm{r}}\right)^{2}$ numbers, and the normalized characteristic indentation dimension vs critical normalized cracking load ratios are plotted in figure $4 \mathrm{~b}$ of [1]. Only some wet and dried materials are named here and the authors of [1] cite hardly 
checkable data collections and unpublished own data (which ones?). For example they did not cite the soda lime glass values from [16], which are still a present ISO-standard for $H_{\mathrm{I}}$ and $E_{\mathrm{I}}$, even though they repeatedly invoked the "Oliver-Pharr analysis" or "-model". And only a few entries are directly cited, but almost none of these disclosed published original loading curves that could be checked and used for the calculation of real properties like physical hardness, iteration-free elastic moduli and phase-transitions under load.

Furthermore, the authors of [1] try to define and calculate a so-called "true hardness" $H_{\text {true }}=H /\left\{1-(H / E)^{1 / 2}(2 / \tan \beta)^{1 / 2}\right\}^{2}$, where $\beta$ is the cone angle of the indenter. This shall be the "resistance to plastic deformation" or "resistance to irreversible deformation", which "depends on the ratio between indentation hardness and indentation modulus". It is strangely claimed that "a large indentation hardness does not imply a large resistance to irreversible deformation per $s e$ ". This hard to understand basis by using the $H / E_{\mathrm{r}}$ fraction is exemplified in [1] as follows: $H=1.07 \mathrm{GPa}$ and $E_{\mathrm{r}}=10.5 \mathrm{GPa}$ shall imply "true hardness" of 17.8 GPa"; or $H=3.12 \mathrm{GPa}$ and $E_{\mathrm{r}}=87.02 \mathrm{GPa}$ shall imply "true hardness" of $10.8 \mathrm{GPa}$ ". The $H / E_{\mathrm{r}}$ fraction is contained in such calculations. And the other formulas are in the appendix of [1]. These need not be depicted here, due to the incorrect physical basis from the beginning. Such "true hardness" with exorbitantly misleading high values adds further to misleading confusion without any physical merit.

\subsection{The Physical Errors of the $H / E$ Ratio Claims with Their Uses}

The proportionality claims of ISO- $H$ with ISO- $E_{\mathrm{r}}$ in [1] and the therein appropriate citations are physically wrong. They cannot be valid for basic physical reasons! The only correct physical hardness of conical or pyramidal indentations is the penetration resistance $k$ [force/depth ${ }^{3 / 2}$ ] from the slope of the so named Kaupp-plot $F_{\mathrm{N}}$ vs $h^{3 / 2}$. None of the cited and used $H_{\mathrm{I}}$ and $E_{\mathrm{I}}$ values tells which polymorph of the material was probed, because their onset forces cannot be found with the wrong exponent 2 on $h$. One needs such linear plots for the detection of polymorph formation onsets [4] [10]-[15]. And every polymorph has its own mechanical properties. With other words:

All of these $H$ and $E_{\mathrm{r}}$ values are unphysical and so are their ratios, because they rely on the disproved exponent 2 on $h$ (instead of the $F_{\mathrm{N}}$ vs $h^{3 / 2}$ relation) and require data-fitting iterations [16]. For correct analysis of loading curves for pyramidal and conical indentations see [3] and [6]. Spherical indentations are more complicated [6], but some of the examples in figure 1 of [1] are from unphysical interpreted spherical indentations or Vickers hardness as in e.g. [17] with similar $H / E$ ratios containing $\log / \log$ plots. Unfortunately, $E_{\mathrm{r}}$ numbers are not well defined and depend strongly on the details of their detection, as outlined in [9], so that one has to choose from sometimes extremely different values for $E_{r}$ when comparing different methods.

The Oliver-Pharr technique and the still present ISO 14577 standard assume 
the physically disproved exponent 2 on $h$ for the loading curves instead of undeniably physically deduced $h^{3 / 2}$ [3], and they violate the energy law [6] [8]. Therefore the authors of [1] cited $H$ and $E_{\mathrm{r}}$ values that are entirely unphysical parameters. If indentation hardness have to be compared with indentation modulus one should only take physically sound values from the so named Kaupp plots ( $F_{\mathrm{N}}$ vs $h^{3 / 2}$ ) that most easily provide penetration resistance onsets and differentiate the properties of every polymorph under load. And it provides directly measured indentation moduli $\left(E_{\text {phys }}\right)$ without any iteration. All of the trouble in Section 3.2 originates from the widespread refusal to check the exponent of their loading curves. These $F_{\mathrm{N}}$ vs $h$ loading curves follow always the physically correct relation $F_{\mathrm{N}}=k h^{3 / 2}$. All of the respective authors stay with the physically disproved $h^{2}$, apparently until ISO and the authors of [16] correct their basic errors with public announcements.

\subsection{The Wealth of Penetration Resistance for the Physical Analysis as Exemplified for the Marine Mollusks Case}

The claims of linear relations between ISO-hardness $H$ and ISO-modulus $E_{\mathrm{r}}$ include nacre, eggshell, aragonite, calcite, hydroxyapatite, enamel, dentine, bone, etc in the unphysical and incorrect log/log plots in [1]. But their data are selective and none of them reveals which polymorph was probed under what applied load. Furthermore, it is not told which of the triangle data points around the regression line in figure 1 of [1] belong to which materials. These data are without any value.

We show now that hitherto unthinkable materials' properties are straightforwardly obtained on the physical analysis of indentations from correctly cited publications. This will be exemplified for the case of two mollusk varieties with their aragonite shells, including the distribution of the organic materials. The physical analysis of the indentation loading curve onto the polar pteropod Limacina Helicina Antarctica shell, as recently published with figure 4 in [2], yields the $F_{\mathrm{N}}$ vs $h^{3 / 2}$ diagram of Figure 1 . One recognizes an initial surface effect up to about $300 \mu \mathrm{N}$ load (probably due to the water content) and three linear branches that are connected by smooth transition zones between them. This was not seen in their $F_{\mathrm{N}}$ vs $h$ curve and it does never show up in the unphysical $F_{\mathrm{N}}$ vs $h^{2}$ relation with its false exponent 2 on $h$. The authors of [2] did thus not see that their calculation of ISO- $H$ and ISO- $E_{\mathrm{r}}$ values [16] do not at all relate to properties of their sample but to the third polymorph of it that is present at their maximal force. Our three linear regression lines with $\left(R^{2}=0.9994,0.9993\right.$, and 0.9993, respectively) have the equations that are inserted in Figure 1. The kink positions, as obtained by equation of two adjacent regression equations, at 1182.08 and $2958.895 \mu \mathrm{N}$ loads are phase-transition onsets. With these experimental values we calculate the phase transition energies with the simple well-known arithmetic equations that are most recently comprehensively published in [6] and [14], and earlier publications of the present author. The obtained normalized per $\mu \mathrm{N}$ values are $0.01496 \mu \mathrm{N} \mu \mathrm{m} / \mu \mathrm{N}$ and $0.14879 \mu \mathrm{N} \mu \mathrm{m} / \mu \mathrm{N}$. Clearly, there 


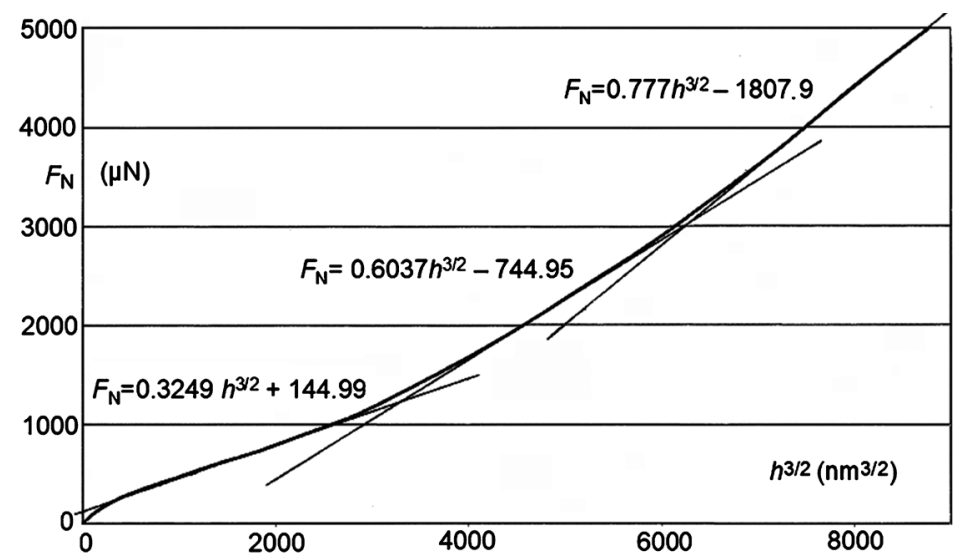

Figure 1. Linear plot of a "typical" Berkovich indentation onto the pteropod Limacina Helicina Antarctica shell with thinner intersecting regression lines, exhibiting an initial surface effect and three straight branches with comparably smooth transition zones between them. The inserted steepness constants $k$ are in $\mu \mathrm{N} / \mathrm{nm}^{3 / 2}$; the original force vs load data are taken from figure 4 in [2].

are three different polymorphs up to a loading range of $5 \mathrm{mN}$ load with a Berkovich. Interestingly these values are similar to the ones of calcite in table 1 of [14] that are at 0.01599 and $0.10692 \mu \mathrm{N} \mu \mathrm{m} / \mu \mathrm{N}$, respectively, although at their $\mathrm{mN}$ ranges. It is clear that we have the phase transitions with the Limacina Helicina Antarctica shell.

The smooth transition zones rather than sharp kinks that are here only seen by the intersecting regression lines reveals a gradual change of the strengthening organic material between the different polymorphs. This is certainly a bionics model for avoiding the crack increasing risk when unavoidable polymorph interfaces contact smoothly [11]. This appears to be further studied and used for modifying the negative effect of phase-transitions also with technical materials.

The precise distribution of the about $5 \mathrm{wt} \%$ of organic material is certainly worth further studies. Conversely, the unphysical $H$ and $E_{\mathrm{r}}$ measurements led to the claim of "essentially homogenous distribution throughout the shell for "strengthening the cell" [2]. Furthermore, the rather strong variation of the averaged ISO- $H$ and ISO- $E_{\mathrm{r}}$ values in figure 5 of [2], or the report that some indentations were going down to $700 \mathrm{~nm}$ depth and others down to only $200 \mathrm{~nm}$ depth, or the observation of rough and smooth areas would also suggest a thorough new and physically analyzed indention. All of these strongly deviating results must be reproduced and separately analyzed rather than averaged as in [2]. Comparison of the so available penetration resistance $k$-values $\left(\mathrm{mN} / \mu \mathrm{m}^{3 / 2}\right)$ of the different polymorphs up to the same load maximum would tell, whether there are zones with more or less organic material also laterally distributed. In the case of micro-caverns empty or filled with water, these would show-up as spurious pop-ins [15]. The physical analysis technique is the only mans for the distinction of soft and hard regions that cannot be visibly traced.

It is particularly unsuitable that the authors of [2] did not compare their findings with the physical analysis of the Berkovich indentation onto the lamellar 
pteropod structure of the red abalone Haliotis rufescens in [5]. This pteropod shell exhibits distinct well defined stepwise organic layers between the aragonite lamellas and the indentation curves of them are physically correct analyzed in [5]. The non-appreciation of the well documented different strengthening bionics model is misleading, even though the introduction of paper [2] cites numerous old and very old papers on different pteropod varieties with prismatic helical and lamellar aragonite structures, but unduly questioned the paper of [18]. We must therefore resume our analyses here with the calculations on the correct physical basis. These add to a much better understanding of the pteropods stability with reliable parameters instead of iterated $H$ and $E_{\mathrm{r}}$ that are against physics.

The averaged Berkovich indentation curve of the red abalone Haliotis rufescens shell from Baja, California [18] was physically analyzed in [14]. The exterior nacre shell of $250-300 \mathrm{~nm}$ thickness with $k_{\text {frrt-aragonite-shell }}=0.9058 \mu \mathrm{N} / \mathrm{nm}^{3 / 2}$ is sharply distinguished by the organic layer with $k_{\text {organic }}=0.274 \mu \mathrm{N} / \mathrm{nm}^{3 / 2}$ and the following inner apatite layer with $k_{\text {inner-aroganite-shell }}=1.1495 \mu \mathrm{N} / \mathrm{nm}^{3 / 2}$. The stepwise behavior is also shown in the original $F_{\mathrm{N}}$ vs $h$ plot from figure 7 in [18]. Clearly both aragonite layers are sharply separated by the soft organic layer. The $F_{\mathrm{N}}$ vs $h^{3 / 2}$ plot in Figure 2 with the inserted regression equations, as calculated up to $400 \mathrm{~nm}$ depth $\left(8000 \mathrm{~nm}^{3 / 2}\right)$ with the inserted regression lines is totally different from Figure 1. After a minor initial surface effect that is not part of the regression ( 5 points) two aragonite layer lines are separated by the organics line. The larger $k$-value of the first inner aragonite layer starts at a 26.9 per cent higher load and at higher $h^{3 / 2}$. Therefore at least some of this $k$-value increase represents the shift relative to the end of the first aragonite layer. The thinner strengthening organic layer is considerably softer. The $16.8 \%$ difference between the $k$-values of the two aragonite layers cannot solely be responsible for their displacement. The second aragonite layer should also be phase-transformed at the increased force with an onset right above the organic layer at $4312 \mu \mathrm{N}$. The regression line values of the hard branches correlate both with $\mathrm{R}^{2}=0.9997$.

However, there are difficulties for the calculation of the phase-transition

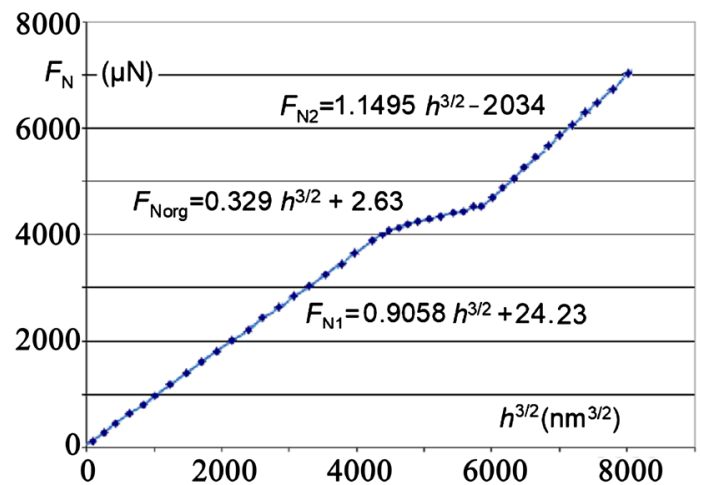

Figure 2. Linear plot of Berkovich indentation onto the red abalone Haliotis rufescens shell from Baja, California [18]; image taken from [5] and with complete inserts that denote the penetration resistance $k$ (their physical hardness $\left(\mu \mathrm{N} / \mathrm{nm}^{3 / 2}\right)$ with the axes cuts of the hard and the soft layers [3] [6]. 
energy, because we do not have a kink-point between the displaced aragonite layers. We must try to secure that the inner aragonite layer is a polymorph by a phase-transition. Figure 2 indicates that the first branch ends very close to the lower end of the organic layer at $4000 \mu \mathrm{N}$ load and $287 \mathrm{~nm}$ depth $\left(4853 \mathrm{~nm}^{3 / 2}\right)$. The second aragonite branch starts directly at the upper end of the organic layer at $4312 \mu \mathrm{N}$. This data point is already part of the regression line for the steeper (harder) branch. The $4000 \mu \mathrm{N}$ load would thus be the phase transition onset point if the organic layer was not there. Unlike the repair of pop-ins [15], our phase-transition energy calculation technique is not applicable for such separation by a different material. A shift of the upper layer line for joining with the lower line at $4000 \mu \mathrm{N}$ by formally removing the organic layer is not allowed. And it would not solve the problem: the steepness of the second hard layer is influenced both by higher $F_{\mathrm{N}}$ and by higher $h^{3 / 2}$ values at its start. This influence on the steepness cannot be undoubtedly judged and also minor corrections in that sense would strongly influence our precise and highly sensitive calculations. A phase transition part from about $4500 \mu \mathrm{N}$ load of the hard nacre shell is however most likely. That judgment is in view of the $k_{1}$ and $k_{2}$ values of the softer Limacina Helicina Antarctica shell that experiences the phase transition and the $k_{1}$ and $k_{2}$ values of the Haliotis rufescens shell that are in the same order of magnitude even though the shells of Limacina Helicina Antarctica are softened by the embedded organic layers. Final proof would require comparison with an indentation of pure aragonite at forces up to about $7000 \mu \mathrm{N}$ load. Unfortunately we did not find accessible reliable Berkovich indentation curves of pure aragonite at such a loading range with smooth loading curves that are not interrupted by continuously repeated unloads. There is however a phase-transition within a 500 $\mu \mathrm{N}$ loading range of pelletized aragonite from [19] that occurs with an endothermic phase-transition at $348 \mu \mathrm{N}$ load We interpret it as an endothermic twinning of aragonite and calculate the normalized conversion energy to 0.02922 $\mu \mathrm{N} \mu \mathrm{m} / \mu \mathrm{N}$. Clearly this interesting twinning transition cannot be remarked at an indentation range of $7000 \mu \mathrm{N}$ of the mollusks indentations.

We must report here how one can identify and exclude experimentally false reported data by the calculation of transition energies. The reported Berkovich indentation onto (001) of aragonite up to $1000 \mu \mathrm{N}$ load from the figure 1 of [20] revealed a minor exothermic transition at $F_{\text {Nkink }}=408.6 \mu \mathrm{N}$ when physically analyzed. Thus, figure 15b in [5] would exhibit exothermic transition energy of $-0.00276 \mu \mathrm{N} \mu \mathrm{m} / \mu \mathrm{N}$ [14]. This putative twinning of aragonite appeared to be a specialty of the (001) face of aragonite. It was hardly resolved in the indentations of nacre up to $7000 \mu \mathrm{N}$ load and must be cancelled now in Table 1 of [14]. This failure is an important application of the calculation of phase-transition energies, because it helps to eliminate undue measurements. The work of [20] failed, because the loading curves were averaged and pop-ins was also reported with three imaged individual curves on the probed surface. Their inclusion in the curves averaging constructed the exothermic event. It is still not widely recog- 
nized that pop-ins are instrumental errors due to distortions that must be repaired or eliminated from further use [15]. The pop-in generation in the present case was probably by touching of tip sides with terrace steps on the probed surface (further reasons of pop-in distortions are listed in [15]). Any averaging of experimental curves must be strictly avoided. Only the results from all individual undistorted curves should be averaged. The curve for (001) in figure 1 of [20] and its analysis in table 1 of [14] must be disregarded.

The behavior of aragonite must now be compared with the other ambient modifications of $\mathrm{CaCO}_{3}$. Hexagonal calcite, orthorhombic aragonite, and hexagonal vaterite crystallize in the respective space groups R3-c, Pbm6n, and $\mathrm{P}_{3} / \mathrm{mmc}$. Their X-ray densities are $2.71,2.93$, and $2.93 \mathrm{~g} / \mathrm{cm}^{3}$, respectively. The most frequent twins of calcite occur along $(10$ - 11) by mechanical stress on $(01$ 12) and those of aragonite on (110) by mechanical stress on (010) [21]. So there is the possibility of mechanical twinning by pressure. Such twins of aragonite are orthorhombic Pmcn. Vaterite twins have been found in pearls [22]. All three modifications occur as minerals. Aragonite and vaterite (with some organic material) are primarily of biological origin as in pearls or mollusk shells, and vaterite in gallstones and nephritic stones and plants (e.g. [23]). An important point is the repair of deformed Gastropodes' aragonite shells with vaterite attachments [23]. The loading curves of calcite up to $10 \mathrm{mN}$ [24] and up to $40 \mathrm{mN}$ [25] have been analyzed in [14] to give conversion energies of $0.01599 \mathrm{mN} \mu \mathrm{m} / \mathrm{mN}$ and $0.1069 \mu \mathrm{N} \mu \mathrm{m} / \mu \mathrm{N}$ for the second phase transition.

\section{Conclusions}

It appears more than surprising that a paper like [1] is still prepared (and can even be published) that rests on disproved historical errors. Since these are against physics, their mechanical parameters required data manipulating iterations and more and more extremely complicated theoretical treatments, as shown here in Section 3.1. Such behavior is still not stopped by the International Standardizing Organization with its ISO 14577. This Organization is very slow with the revision of their standards that still enforce industries for being certified. It did not yet help that the easily empirically found (since 2004) [4] and straightforwardly deduced (convincingly published in 2016 [3] and in 2020 enhanced [6]) physical equations can be simple reproduced. They are successfully published and provide numerous unprecedented arithmetic applications. But influential people, who stick to history and did or taught it always historically, are restrictive. Unfortunately, the false characterization of mechanical properties is continuously leading to catastrophic disasters. But for perhaps obvious responsibility reasons, it is not tried to assign the reasons for these disasters to poor alloys in need of improvement for airplanes, for public bridges, and all further construction materials. Upon catastrophic events, one just refers to obeying ISO standards and tries for example in the case of continuing airliner crashes only to blaming pilot errors or deficient piloting control software, but not also to unfit 
physically incorrectly analyzed materials. Thus, indentations revealing phasetransition onset control of the materials upon mechanical (cf. Figure 1) and thermal (cf. [12]) stress are disregarded, ignored, or denied. But why not avoid braking turbine propeller blades with improved alloys that withstand higher forces before phase-transition onsets occur [14]. We also complain the cracking between wing and fuselage (pickle fork) and hundreds of grounded airliners with multiple clefts on fuselage, etc. These are materials' failures. Pickle forks must not just be repaired, but constructed with improved alloys and replaced. The checking and improving of the alloys must be for increasing the phasetransition onsets forces and energies. Polymorph interfaces from phase transitions are sites for cracks' nucleation with catastrophic failures [11] [12] [13] [15]. The detection of phase transitions under load is still not part of ISO 14577, because it cannot achieve them by using the false exponent 2 on $h$ instead of $3 / 2$ from indentation loading curves.

An $H / E_{\mathrm{r}}$ ratio (also called "elasticity index") is unphysical, as are ISO- $H$ and ISO- $E_{\mathrm{r}}$. Physical hardness is $H_{\text {phys }}=k / \pi \tan \alpha^{2}$ ( $k$ in force/depth ${ }^{3 / 2}, \alpha$ effective cone angle) for conical and pyramidal indenters. And the not iterated physical modulus is $E_{\mathrm{rphys}}=S / 2 h_{\max } \tan \alpha$ as deduced in [9]. The intrinsic forms (for only volume formation) require the factor 0.8 on $F_{\mathrm{N} \max }$ [8] [26] for obeying with the energy law. Modern instrumentation provides enough data points for the initial linear slope that is stiffness $S$ of the unloading curve. The assumed but not physically deduced linearity between $H$ and $E_{\mathrm{r}}$ has not been demonstrated by the log-log plot in figure 1 of [1] with a "statistical confidence of $95 \%$ " and $\mathrm{R}^{2}=0.96$ for the selected materials by not considering their undetermined phase-transitions that are however most frequent for all kinds of materials upon load. Even under these unsuitable conditions the actual deviations are very often enormous. However, most materials have to be again indented when neither original data, nor published $F_{\mathrm{N}}$ vs $h$ loading curves had been published. ISO- $H$ and ISO- $E_{\mathrm{r}}$ values cannot reinstall the physical indentation results, due to the exhaustive data fitting iterations. It has to be rejected that the $H / E_{\mathrm{r}}$ ratio is used for defining a so-called "true hardness" with extremely high useless values of hardness and moduli. Also the revival of the complicated formulas, using $H / E_{\mathrm{r}}$ ratios for brittle parameter, yield strength, and toughness, is misleadingly incorrect and useless. Correct unprecedented qualities of materials (as exemplified in Section 3.3.) are to be deduced from a physically sound basis. The easily obtained experimental achievements on the basis of the physical analyses of indentations are withheld in [1] from its audience of biologists, pharmacists, physicians, theoreticians, and other readers.

The exemplarily analysis of the indentations onto seawater mollusks shows that ISO- $H$ and ISO- $E$ values are unable to differentiate between the construction principles of different mollusk shells. The aims to solve important biological questions are not attained and so are the theoretical speculations. It requires the so named Kaupp-plot ( $F_{\mathrm{N}}$ vs $h^{3 / 2}$ ) for most easily and rewardingly revealing the striking differences. In the case of Limacina Helicina Antarctica the linearized 
loading curve undergoes two phase-transitions in the load ranges up to $5 \mathrm{mN}$. The within aragonite distributed material cushions shocks so that the shells are protected. We quantified the phase-transition onsets and energies that reflect the details for the lattice conversion in Section 3.3. A new bionics model is extracted from the shell behavior. Its cell strength is achieved by mitigation of the dangerous effects of polymorph interfaces by softening with gradual approach to polymorphs interface from the unavoidable phase-transitions. This bionics model should become most useful for technical materials that are exposed to mechanical forces that induce phase-transition onsets in e.g. ballistics or earthquakes etc. Furthermore, these results open up new technical and biological insights. Further indentations onto Limacina Helicina Antarctica, as requested in Section 3.3 will in the future facilitate the crystallographic understanding of these phase-transitions with eventually further bionics models.

Totally different is the already known bionics model of Haliotis rufescens. It uses alternating layers of the thin soft organic material between thicker aragonite layers for cushioning. Also further studies with the numerous further mollusks become worthwhile and promising now. Variations of the layer thickness and detailed structures in the not layered varieties with respect to environmental conditions will provide biological answers. Also snail-shell indentations should be physically analyzed, but not with the disproved and unable techniques in figures 1 and 2 of [1], as discussed in Section 2.

Further advances of the physical analyses, in addition to the precise detection of phase-transition onset forces and energies for explaining and avoiding catastrophic failures, are the sorting out of initial surface effects, the detection and elimination of experimentally false reports with the calculation of phase-transition energies and the distinction of phase-transition onsets from those of different material layers. When measured at various temperatures one can also calculate the activation energies of phase-transitions [12]. None of these achievements are available from $H, E_{\mathrm{r}}, H / E_{\mathrm{r}}$ and there from deduced obsolete techniques, because they are all unphysical.

The future will detect and understand further phase-transition onsets and energies from all kinds of materials. This opens their discussion for the widening of their understanding and applications, and the search for further bionics models appears promising.

It is hoped that not only biologists, pharmacists, physicians, and also industrial Engineers take their chance to increase and revise their knowledge for preventing dangerous disasters by using the penetration resistance instead of ISO- $H$ and ISO- $E_{\mathrm{r}}$. The revision of ISO-14577 must also be accelerated. It is strongly hoped that ISO 14577 and the authors of [16] correct their basic errors with public announcements as soon as possible for a safer world.

\section{Conflicts of Interest}

The author declares no conflicts of interest regarding the publication of this paper. 


\section{References}

[1] Labonte, D., Lenz, A.K. and Oyen, M.L. (2017) On the Relationship between Indentation Hardness and Modulus, and the Damage Resistance of Biological Materials. Acta Biomaterialia, 57, 373-383. https://doi.org/10.1016/j.actbio.2017.05.034

[2] Teniswood, C.M.H., Roberts, D., Howard, R. and Bradby, J.E. (2013) A Quantitative Assessment of the Mechanical Strength of the Polar Pteropod Limacina Antarctica Shell. Journal of Marine Science, 70, 1499-1505.

https://doi.org/10.1093/icesjms/fst100

[3] Kaupp, G. (2016) The Physical Foundation of $F_{\mathrm{N}}=k h^{3 / 2}$ for Conical/Pyramidal Indentation Loading Curves. Scanning, 38, 177-179. https://doi.org/10.1002/sca.21223

[4] Kaupp, G. and Naimi-Jamal, M.R. (2004) Nanoscratching on Surfaces: The Relationships between Lateral Force, Normal Force and Normal Displacement. Zeitschrift für Metallkunde. International Journal of Materials Research and Advanced Techniques, 95, 297-305.

[5] Kaupp, G. (2012) Penetration Resistance and Penetrability in Pyramidal Nanoindentations. Scanning, 35, 88-111. https://doi.org/10.1002/sca.21038

[6] Kaupp, G. (2020) Valid Geometric Solutions for Indentations with Algebraic Calculations. Advances in Pure Mathematics, 10, 322-336. (Erratum (2020) Advances in Pure Mathematics, 10, 545-546.) https://doi.org/10.4236/apm.2020.105019 https://doi.org/10.4236/apm.2020.109034

[7] Kaupp, G. (2013) Penetration Resistance: A New Approach to the Energetics of Indentations. Scanning, 35, 392-401. https://doi.org/10.1002/sca.21080

[8] Kaupp, G. (2017) The ISO Standard 14577 for Mechanics Violates the First Energy Law and Denies Physical Dimensions. Journal of Material Sciences \& Engineering, 6, 321-328. https://doi.org/10.4172/2169-0022.1000321

[9] Kaupp, G. (2017) Dilemma between Physics and ISO Elastic Indentation Modulus. Journal of Material Sciences and Engineering, 6, 402-405.

https://doi.org/10.4172/2169-0022.1000402

[10] Kaupp, G. and Nami-Jamal, M.R. (2010) The Exponent 3/2 at Pyramidal Nanoindentations. Scanning, 32, 265-281. https://doi.org/10.1002/sca.20206

[11] Kaupp, G. (2018) Six Polymorphs of Sodium Chloride upon Depth Sensing Macroindentation with Unusual Long-Range Cracks Requiring 30 N Load. Journal of Material Sciences and Engineering, 7, 473-483.

https://doi.org/10.4172/2169-0022.1000473

[12] Kaupp, G. (2014) Activation Energy of the Low-Load NaCl Transition from Nanoindentation Loading Curves. Scanning, 36, 582-589.

https://doi.org/10.1002/sca.21158

[13] Kaupp, G. (2019) Phase-Transition Energies, New Characterization of Solid Materials and Anisotropy. Advances in Materials Physics and Chemistry, 9, 57-70. https://doi.org/10.4236/ampc.2019.94006

[14] Kaupp, G. (2019) Physical Nanoindentation: From Penetration Resistance to PhaseTransition Energies. Advances in Materials Physics and Chemistry, 9, 103-122. https://doi.org/10.4236/ampc.2019.96009

[15] Kaupp, G. (2020) Indentation onto Stishovite $\left(\mathrm{SiO}_{2}\right), \mathrm{MgO}$, and a Covered Superalloy: "Pop-In" Repair, Phase-Transition Onsets, Polymorph Energies, and Transition-Energies. Advances in Materials Physics and Chemistry, 10, 77-95. https://doi.org/10.4236/ampc.2020.103007

[16] Oliver, W.C. and Pharr, G.M. (1992) An Improved Technique for Determining 
Hardness and Elastic Modulus Using Load and Displacement Sensing Indentation Experiments. Journal of Materials Research, 7, 1564-1583. https://doi.org/10.1557/JMR.1992.1564

[17] Rhee, Y.W., Kim, H.W., Deng, Y. and Lawn, B.R. (2001) Brittle Fracture versus Quasi Plasticity in Ceramics: A Simple Predictive Index. Journal of the American Ceramic Society, 84, 561-565. https://doi.org/10.1111/j.1151-2916.2001.tb00698.x

[18] Katti, K.S., Mohanty, B. and Katti, D.R. (2006) Nanomechanical Properties of Nacre. Journal of Materials Research, 21, 1237-1242. https://doi.org/10.1557/jmr.2006.0147

[19] Sevcik, R., Sasek, P. and Viani, A. (2018) Physical and Nanomechanical Properties of the Synthetic Anhydrous Crystalline $\mathrm{CaCO}_{3}$ Polymorphs: Vaterite, Aragonite and Calcite. Journal of Materials Science, 53, 4022-4033. https://doi.org/10.1007/s10853-017-1884-x

[20] Kearney, C., Zhao, Z., Bruet, B.J.F., Radovitky, R., Boyce, M.C. and Ortiz, C. (2006) Nanoscale Anisotropic Plastic Deformation in Single Crystal Aragonite. Physical Review Letters, 96, Article ID: 255505. https://doi.org/10.1103/PhysRevLett.96.255505

[21] Anthony, J.W., Bideaux, R.A., Bladh, K.W. and Nichols, M.C. (Eds.) (2003) Handbook of Mineralogy. Mineralogical Society of America, Vol. 5. https://www.handbookofmineralogy.org

[22] Qiao, L. and Feng, Q.L. (2007) Study on Twin Stacking Faults in Vaterite Tablets of Freshwater Lacklustre Pearls. Journal of Crystal Growth, 304, 253-256. https://doi.org/10.1016/j.jcrysgro.2007.02.001

[23] Spann, N., Harper, E.M. and Aldridge, D.C. (2010) The Unusual Mineral Vaterite in Shells of the Freshwater Bivalve Corbicula fluminea from the UK. Naturwissenschaften, 97, 743-751. https://doi.org/10.1007/s00114-010-0692-9

[24] Guillonneau, G., Kermouche, G.K., Bec, S. and Loubet, J.L. (2012) Determination of Mechanical Properties by Nanoindentation Independently of Indentation Depth Measurement. Journal of Materials Research, 27, 2551-2560. https://doi.org/10.1557/jmr.2012.261

[25] Presser, V., Gerlach, K., Vohrer, A., Nickel, K.G. and Dreher, W.F. (2010) Determination of the Elastic Modulus of Highly Porous Samples by Nanoindentation: A Case Study on Sea Urchin Spines. Journal of Materials Science, 45, 2408-2418. https://doi.org/10.1007/s10853-010-4208-y

[26] Kaupp, G. (2020) Real and Fitted Spherical Indentations. Advances in Materials Physics and Chemistry, 10, 207-228. https://doi.org/10.4236/ampc.2020.1010016 